AperTO - Archivio Istituzionale Open Access dell'Università di Torino

Screening of depression in cardiology: A study on 617 cardiovascular patients

This is a pre print version of the following article:

Original Citation:

Availability:

This version is available http://hdl.handle.net/2318/1645522

since 2017-12-13T19:43:28Z

Published version:

DOI:10.1016/j.ijcard.2017.07.065

Terms of use:

Open Access

Anyone can freely access the full text of works made available as "Open Access". Works made available under a Creative Commons license can be used according to the terms and conditions of said license. Use of all other works requires consent of the right holder (author or publisher) if not exempted from copyright protection by the applicable law. 
This Accepted Author Manuscript (AAM) is copyrighted and published by Elsevier. It is posted here by agreement between Elsevier and the University of Turin. Changes resulting from the publishing process - such as editing, corrections, structural formatting, and other quality control mechanisms - may not be reflected in this version of the text. The definitive version of the text was subsequently published in INTERNATIONAL JOURNAL OF CARDIOLOGY, None, 2017, 10.1016/j.ijcard.2017.07.065.

You may download, copy and otherwise use the AAM for non-commercial purposes provided that your license is limited by the following restrictions:

(1) You may use this AAM for non-commercial purposes only under the terms of the CC-BY-NC-ND license.

(2) The integrity of the work and identification of the author, copyright owner, and publisher must be preserved in any copy.

(3) You must attribute this AAM in the following format: Creative Commons BY-NC-ND license (http://creativecommons.org/licenses/by-nc-nd/4.0/deed.en), 10.1016/j.ijcard.2017.07.065

The publisher's version is available at:

http://linkinghub.elsevier.com/retrieve/pii/S0167527317315024

When citing, please refer to the published version.

Link to this full text:

http://hdl.handle.net/2318/1645522 


\section{Screening of depression in cardiology: a study on 617 cardiovascular patients.}

Valentina Tesio ${ }^{1,2}$, Sebastiano Marra ${ }^{1,3}$, Stefania Molinaro ${ }^{1,4}$, Riccardo Torta ${ }^{1,4}$, Fiorenzo Gaita $^{1,3}$, Lorys Castelli ${ }^{1,2}$.

Corresponding author: Tesio Valentina; email: valentina.tesio@unito.it.

c/o Department of Psychology, University of Turin, Via Po 14, 10123 Turin, Italy;

Conflict of Interest: none declared.

Key words: cardiovascular disease; depression; coronary artery disease; acute coronary syndrome; HADS; screening.

${ }^{1}$ This author takes responsibility for all aspects of the reliability and freedom from bias of the data presented and their discussed interpretation.

${ }^{2}$ Department of Psychology, University of Turin, Via Po 14, 10123, Turin, Italy.

${ }^{3}$ Division of Cardiology, University of Turin, "Città della Salute e della Scienza" Hospital, Corso Bramante 88, 10126, Turin, Italy.

${ }^{4}$ Clinical Psychology and Psycho-Oncology Unit, University of Turin, "Città della Salute e della Scienza” Hospital, Corso Bramante 88, 10126, Turin, Italy. 


\section{Abstract \\ Background}

Depression screening in the cardiovascular disease (CVD) care setting is under-performed, also because the issue of the optimal screening tools cut-off is still open. We analysed which HADS (Hospital Anxiety and Depression Scale) total score cut-off value shows the best properties in two groups of 357 Acute Coronary Syndrome (ACS) and 260 Chronic Coronary Artery Disease (CAD) hospitalized patients.

\section{Methods}

A Receiver Operating Characteristics (ROC) curve was plotted for both groups using the Montgomery-Asberg Depression Rating Scale (MADRS) as the criterion. Accuracy, positive (PPV) and negative (NPV) predictive values were computed for different cut-off scores.

\section{Results}

The ROC curves confirmed the excellent/very good accuracy of the HADS in both groups, with an area under the curve of .911 for the ACS and .893 for the CAD patients. The cut-off of 14 showed the best compromise between high sensitivity and good specificity in both groups, with high negative predicted values (95.5\% and $92.4 \%$, respectively).

\section{Conclusion}

Using a cut-off value of 14 , the HADS could be considered a good screening tool to identify hospitalized CAD and ACS patients requiring a more accurate depression assessment, in order to promptly plan the most appropriate treatment strategies and prevent the negative effects of depression in CVD patients. 


\section{Introduction}

A recent systematic analysis of the "Global Burden of Disease Study 2013" underlined once again that cardiovascular deaths still account for almost a third of all deaths globally [1]. There is extensive evidence that cardiovascular disease (CVD) and depression reinforce each other, with a downward spiral association [2]. Depression results in an $80-90 \%$ increased risk of CVD onset [3] and is strongly linked with adverse outcomes, negatively contributing to the progression and prognosis of the disease. Furthermore, CVD itself could increase the risk of developing depressive disorders [2], with Major Depressive Disorder (MDD) showing a prevalence of $20 \%$ to $30 \%$ in coronary heart disease (CHD) patients [4].

Although several clinical guidelines recommend early screening and evaluation for treatment of depression [5], clinical cardiologists often do not take into account depression as a negative risk factor, underestimating its negative effect. What is more, routine screening in the hospital care setting is still under-performed, also because of conflicting and confused findings regarding the optimal tool $[4,6,7]$. A recent systematic review analysed the diagnostic accuracy of different screening tools, including the "Hospital Anxiety and Depression Scale" (HADS), in detecting MDD in CHD patients [4]. Although it highlighted that the HADS is a useful self-report instrument in CVD patients [4], other studies call into question the diagnostic accuracy of the two subscales separately, but strongly suggest the use of the HADS total score (HADS-Tot) [8-11]. On these bases, the general aim of this study is to provide clinicians with the optimal HADS cut-off to identify depressed patients. We thus investigated the concurrent validity of the HADSTot with a clinician-rated scale, assessing depression in two groups of cardiac hospitalized patients - Acute Coronary Syndrome (ACS) and chronic Coronary Artery Disease (CAD). 


\section{Methods}

\subsection{Patients and procedure}

The study was proposed to all consecutive patients hospitalized in the Cardiology Unit of the "Città della Salute e della Scienza" Hospital of Turin. All patients who gave their written informed consent and completed the HADS had a clinical interview with an expert psychologist who filled in the Montgomery-Asberg Depression Rating Scale (MADRS). From a pool of 1049 inpatients who completed the HADS, the data of 357 patients with ACS and 260 with chronic CAD were analysed.

\subsection{Measures}

The MADRS, a semi-structured clinical interview rated by an expert clinical psychologist/psychiatrist, is one of the gold-standard clinician-rated diagnostic tools [12]. Following the widely-accepted recommendations, the cut-off value of 11 was used to tally a patient as depressed $(\geq 11)$ or not $(<11)[12,13]$.

The HADS is a brief self-rating instrument composed of 14 items rated between 0-3, widely used in physically ill patients $[14,15]$. It evaluates the presence of depressive and anxiety symptoms. According to the more recent methodological studies published on the matter, the total score was used as an index of psychological distress [9].

\subsection{Statistical analyses}

All statistical analyses were performed using the Statistical Package for Social Science (SPSS-23). A Receiver Operating Characteristics (ROC) curve was plotted separately for ACS and CAD patients, using the results of the MADRS as the criterion.

Positive (PPV) and negative (NPV) predictive values, accuracy and distance (d) to the optimal 
$(0,1)$ point were computed for different cut-off scores. To determine the optimal cut-off score, both the "point of curve closest to the $(0,1)$ " criteria (d value) and visual assessment of the score combining maximal sensitivity with optimal specificity were considered.

\section{Results}

Table 1 shows the demographic characteristics of the two groups. ACS and CAD patients reported a mean (SD) HADS-Tot raw score of 10.7 (7.5) and 11.8 (7.4) respectively, with 19\% (68) of patients in the former group and $29.6 \%$ (77) in the latter showing clinically relevant depressive symptoms according to the MADRS (Table 1).

The ROC curve analyses showed an excellent/very good accuracy of the HADS-Tot in both groups, with an area under the curve (AUC) of .911 for the ACS and .893 for the CAD group

(Table 2). Between the possible cut-off values with the lowest $d$ values, visual inspection of the data revealed that the cut-off of 14 showed the best compromise between high sensitivity and good specificity in both ACS and CAD patients, with high NPVs (95.5\% for ACS and 92.4\% for CAD) (Table 2).

\section{Discussion}

Depressive symptoms have a negative role in CVD, increasing the risk of new cardiac events and negative outcomes, and worsening patients' quality of life [2]. Indeed, post-myocardial infarction (MI) depression is associated with a 1.5- to 2.7-fold increased risk for both post-MI mortality and new cardiac events [16]. Despite the negative effects of depression on cardiac patients, its assessment is still underused, especially in the Italian cardiology hospital setting. 
The opportunity to screen for and treat depression in hospitalized cardiac patients should not be missed, also because the risk of mortality or new cardiovascular events in cardiac patients with depression increases mainly in the first two years after the original cardiac event [17].

Accurate assessment of depressive disorders with a semi-structured interview, such as the Structured Clinical Interview for DSM disorders (SCID-I) or the MADRS, would be costly for the healthcare system in terms of both time (more than 30 minutes) and money, and may not suit the routine of the hospital setting. This is why clinical guidelines $[5,18]$ usually recommend a two-step procedure: first, screening with a self-report scale; then, evaluation with a more accurate assessment (i.e. MADRS) of positive patients. So it is crucial to choose a rapid self-administered tool allowing easy but accurate first-step screening, in order to accurately identify those patients requiring further psychological assessment. The HADS is a clinical tool specifically developed to assess depressive and anxiety symptoms in physically ill patients [19]. It has the advantage of being an easy and rapid self-report instrument (no more than 5 minutes) that can be administered during admission or in a waiting room and takes no longer than a minute to be scored by the clinician. The HADS screening procedure could easily be inserted into the hospital routine, allowing the screening of all patients and reducing drop-out, since discharged patients are often unwilling to return for further scheduled medical appointments. To this aim, recent evidence suggests using the HADS total score, but not the two subscales score separately [8-11]. This indication has both a clinical and a methodological rationale. Clinically, depressive disorders are often the result of not only depressive but also anxiety symptoms, which frequently overlap. Indeed, symptoms of anxiety and depression could both be considered parts of that multidimensional continuum called psychological distress, 
which ranges from normal feelings of vulnerability, sadness and fears to problems that could become disabling, such as depressive disorders. A valid first-step screening tool should therefore account for the overall psychological distress [11].

From a methodological standpoint, recent confirmatory factor analysis (CFA) suggests that the HADS is saturated by a single general distress factor (explaining over $70 \%$ of the variance), with the two subscales showing weak item loadings separately $[9,10]$. These data provide psychometric evidence against the internal consistency of the two subscales in favor of the reliability of the HADS-Tot $[9,10]$. What is more, a recent study shows that the HADS-Tot is a valid measure and that it is predictive of future symptoms of distress, healthcare costs and quality of life in a CHD population [11].

The data of the present study show that, compared to the MADRS, the HADS-Tot has good reliability and accuracy for ACS and CAD patients. In both groups, 14 is the cut-off with the best psychometric properties to screen those patients with clinically relevant levels of psychological distress who should be submitted to a more in-depth assessment for depression. This cut-off provides not only high sensitivity, but also high specificity, with about 4 out of 5 patients correctly classified, as shown by the accuracy values. What is more, this cut-off shows high NPVs (95\% for ACS and 92\% for CAD), meaning that 9 out of 10 patients who, according to the HADS-Tot did not present a clinically relevant level of psychological distress and therefore did not require further psychological investigation, were correctly identified (i.e. they really were non-depressed, according to the MADRS). Clinically, these screening procedures allow the healthcare provider to correctly reduce the number of patients requiring more in-depth depression assessment procedures (clinical interviews) with a considerable 
reduction in time and money spent. What is more, using a unique cut-off for both pathologies could increase the practicality of this tool, allowing an immediate scoring of the HADS, independently of the cardiac diagnosis.

In conclusion, the data of the present study suggest that, using a cut-off value of 14, the HADS could be considered a good first-step screening tool in order to identify CAD and ACS inpatients with a relevant level of psychological distress, who risk developing depressive disorders, and thus require a deeper second-step assessment. 


\section{REFERENCES}

[1] GBD 2013 Mortality and Causes of Death Collaborators. Global, regional, and national age-sex specific all-cause and cause-specific mortality for 240 causes of death, 19902013: a systematic analysis for the Global Burden of Disease Study 2013. Lancet. 2015; 385(9963): 117-71. doi: 10.1016/S0140-6736(14)61682-2.

[2] Penninx BW. Depression and cardiovascular disease: Epidemiological evidence on their linking mechanisms. Neurosci Biobehav Rev. 2016. pii: S0149-7634(15)30355-9. doi: 10.1016/j.neubiorev.2016.07.003. [Epub ahead of print]

[3] Nicholson A, Kuper H, Hemingway H. Depression as an aetiologic and prognostic factor in coronary heart disease: a meta-analysis of 6362 events among 146538 participants in 54 observational studies. Eur. Heart J. 2006; 27:2763-2774.

[4] Ren Y, Yang H, Browning C, Thomas S, Liu M. Performance of screening tools in detecting major depressive disorder among patients with coronary heart disease: a systematic review. Med Sci Monit. 2015; 21:646-53. doi: 10.12659/MSM.892537.

[5] Lichtman JH, Bigger JT Jr, Blumenthal JA, et al. Depression and coronary heart disease: recommendations for screening, referral, and treatment: a science advisory from the American Heart Association Prevention Committee of the Council on Cardiovascular Nursing, Council on Clinical Cardiology, Council on Epidemiology and Prevention, and Interdisciplinary Council on Quality of Care and Outcomes Research: endorsed by the American Psychiatric Association. Circulation. 2008; 118(17): 1768-75. 
[6] Ceccarini M, Manzoni GM, Castelnuovo G. Assessing depression in cardiac patients: what measures should be considered? Depress Res Treat. 2014; 2014:148256. doi: $10.1155 / 2014 / 148256$.

[7] Tesio V, Molinaro S, Castelli L, et al. Screening of depression in cardiac patients: is the HADS an effective tool? Int J Cardiol. 2014; 172(2):e344-5. doi:

10.1016/j.ijcard.2013.12.308.

[8] Cosco TD, Doyle F, Ward M, McGee H. Latent structure of the Hospital Anxiety And Depression Scale: A 10-year systematic review. J Psychosom Res 2012;72:180-4.

[9] Norton S, Cosco T, Doyle F, Done J, Sacker A. The Hospital Anxiety and Depression Scale: A meta confirmatory factor analysis. J Psychosom Res 2013;74:74-81.

[10] Burns A, Hofer S, Curry P, Sexton E, Doyle F (2014) Revisiting the dimensionality of the Hospital Anxiety and Depression Scale in an international sample of patients with ischaemic heart disease. J Psychosom Res 77: 116-121. doi:

10.1016/j.jpsychores.2014.05.005 PMID: 25077852

[11] Palacios JE, Khondoker M, Achilla E, Tylee A, Hotopf M. A Single, One-Off Measure of Depression and Anxiety Predicts Future Symptoms, Higher Healthcare Costs, and Lower Quality of Life in Coronary Heart Disease Patients: Analysis from a Multi-Wave, Primary Care Cohort Study. PLoS One. 2016 Jul 27;11(7):e0158163. doi: 10.1371/journal.pone.0158163.

[12] Montgomery S. Clinically relevant effect sizes in depression. European Neuropsychopharmacology 1994; 4: 283-4. 
[13] Schulte-van Maaren YW, Carlier IV, Zitman FG, et al. Reference values for major depression questionnaires: the Leiden Routine Outcome Monitoring Study. J Affect Disord. 2013; 149(1-3): 342-9. doi: 10.1016/j.jad.2013.02.009.

[14] Zigmond AS, Snaith RP. The Hospital Anxiety and Depression Scale. Acta Psych Scand 1983; 67: 361-70.

[15] Bambauer KZ, Locke SE, Aupont O, Mullan MG, McLaughlin TJ. Using the Hospital Anxiety and Depression Scale to screen for depression in cardiac patients. General Hospital Psychiatry 2005; 27: 275-284.

[16] Meijer A, Conradi HJ, Bos EH, Thombs BD, van Melle JP, de Jonge P. Prognostic association of depression following myocardial infarction with mortality and cardiovascular events: a meta-analysis of 25 years of research. Gen Hosp Psychiatry, 2011; 33:203-16.

[17] van Melle JP, de Jonge P, Spijkerman TA, et al. Prognostic association of depression following myocardial infarction with mortality and cardiovascular events: a metaanalysis. Psychosom Med 2004; 66:814- 822.

[18] Jneid H, Anderson JL, Wright RS, et al. 2012 ACCF/AHA Focused Update Incorporated Into the ACCF/AHA 2007 Guidelines for the Management of Patients with Unstable Angina/Non-ST-Elevation Myocardial Infarction: A Report of the American College of Cardiology Foundation/American Heart Association Task Force on Practice Guidelines. $J$ Am Coll Cardiol. 2013; 61(23): e179-347. doi: 10.1016/j.jacc.2013.01.014. 
[19] Bjelland I, Dahl AA, Tangen Haug T, Neckelmann D. The validity of the Hospital Anxiety and Depression Scale: An updated literature review. Journal of Psychosomatic Research 2002; 52: 69-77. 
Table 1. Demographic and clinical characteristics of the 617 CVD patients.

\begin{tabular}{|c|c|c|}
\hline Variable & $\operatorname{ACS}(\mathbf{N}=357)$ & CAD $(\mathrm{N}=260)$ \\
\hline Age (Mean (SD)) & $67.3(11.4)$ & $69(9.8)$ \\
\hline \multicolumn{3}{|l|}{$\operatorname{Sex}(N(\%))$} \\
\hline Male & $269(75.4)$ & $190(73.1)$ \\
\hline Female & $88(24.6)$ & $70(26.9)$ \\
\hline Educational level (Mean (SD)) & $9.6(4.4)$ & $9.7(4.1)$ \\
\hline \multicolumn{3}{|l|}{ Marital status $(N(\%))$} \\
\hline Single & $25(7)$ & $18(6.9)$ \\
\hline Married/cohabiting & $264(74.4)$ & $200(76.9)$ \\
\hline Divorced & $21(5.9)$ & $21(8.1)$ \\
\hline Widowed & $45(12.7)$ & $21(8.1)$ \\
\hline \multicolumn{3}{|l|}{ Employment status $(N(\%))$} \\
\hline Employed & $97(29.3)$ & $50(19.9)$ \\
\hline Unemployed/homemaker & $22(6.7)$ & $22(8.8)$ \\
\hline Retired & $212(64)$ & $179(71.3)$ \\
\hline MADRS & $6.8(5.9)$ & $8.6(6.2)$ \\
\hline Above cut-off (Cases) (N (\%)) & $68(19)$ & $77(29.6)$ \\
\hline HADS-Tot & $10.7(7.5)$ & $11.8(7.4)$ \\
\hline HADS-D & $4.9(4.2)$ & $5.7(4.1)$ \\
\hline HADS-A & $5.8(4.2)$ & $6.2(4.1)$ \\
\hline
\end{tabular}

ACS: Acute Coronary Syndrome; CAD: chronic Coronary Artery Disease;

MADRS: Montgomery-Asberg Depression Rating Scale;

HADS-Tot: Hospital Anxiety and Depression Scale - total score;

HADS-D/-A: Hospital Anxiety and Depression Scale - depression/anxiety subscale. 


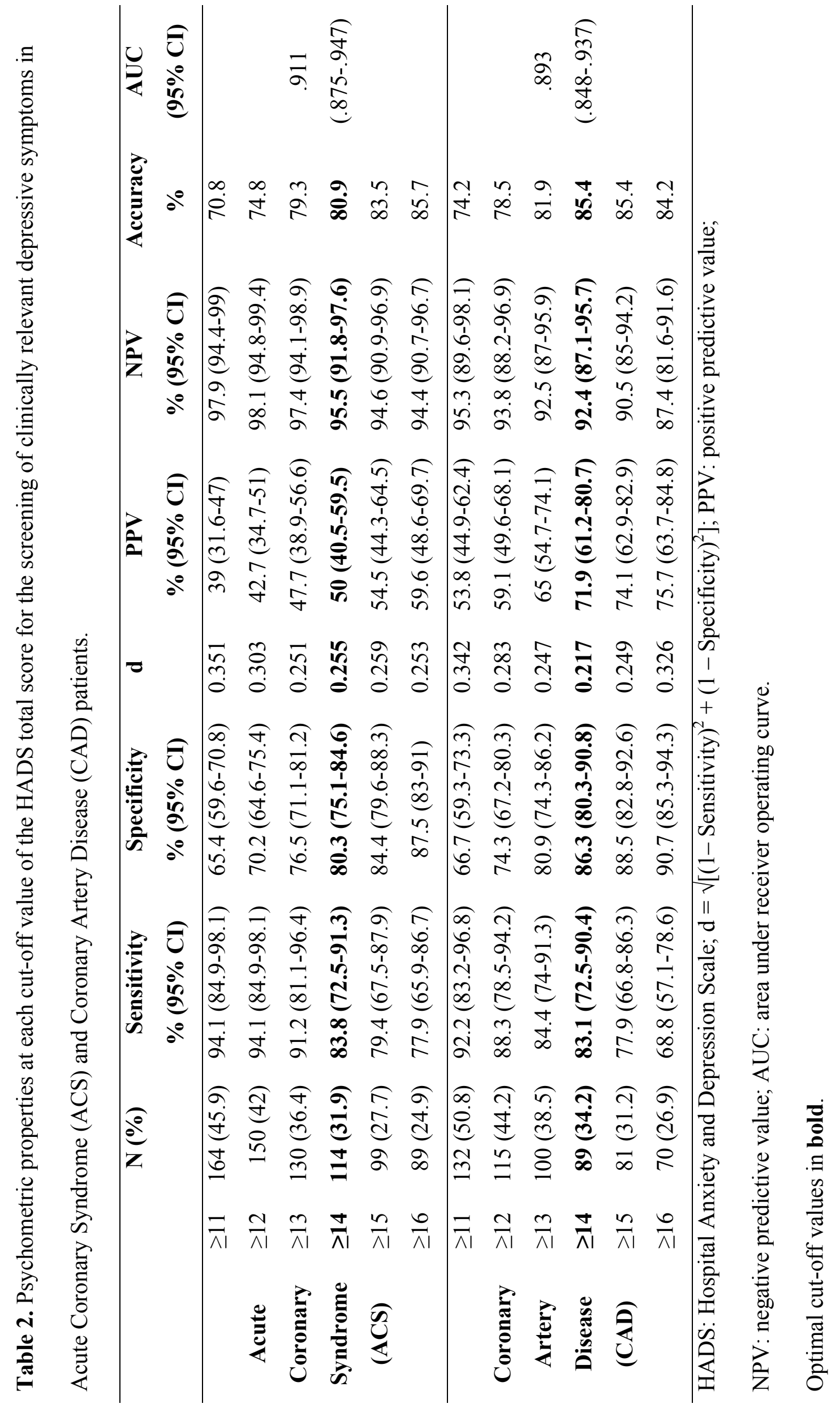

